\title{
Detection and Identification of Single Molecules
}

DOI: $10.1134 / \mathrm{S} 1061934809120016$

Chemistry of single molecules is a new field of research; its existence twenty years ago seemed impossible. Now this direction not only exists but is thought of as an advanced one [1-3]. Analytical chemistry of single molecules is also being developed: these can be detected, identified, and counted, molecule by molecule. This became possible because of the appearance and constant sophistication of analytical methods.

Chemists can excite and register luminescence of single molecules of organic substances, record electronic and vibrational spectra of molecules or estimate their electric conductivity. An important role is played by the modern methods of nanotechnology, including scanning tunnel microscopy, which under certain conditions acts as scanning tunnel spectroscopy. Many similar facts are considered in the big review [1]; other examples can be found in recent publications [4-7]. Thus, nanosize devices based on one-wall carbon nanotubes (CNT) are sensitive to single molecules, in particular hydrogen peroxide and melphalan (alkylating agent) [4]. The adsorption of even one molecule of a certain chemical compound results in a change in the photoluminescence spectra of the device. A DNA molecule is attached to a CNT, and the CNT becomes a biosensor. Upon its contact with a melphalan molecule, peaks in the luminescence spectrum shift to the red, and the hydrogen peroxide molecule reduces the intensity of its luminescence. Analysts can detect peroxide molecules, because the intensity of photoluminescence changes stepwise, with each molecule of hydrogen peroxide.

DNA molecules can be counted with high efficiency in a microfluid flow system [5]. Glass nanopores incorporating cyclodextrin allow analysts to "catch" single molecules of optical isomers [7].

\section{REFERENCES}

1. Buchachenko, A.L., Usp. Khim., 2006, vol. 75, no. 1, p. 3.

2. Science, 2007, May 25.

3. Murrey, R.W., Anal. Chem., 2007, vol. 79, no. 13, p. 4739.

4. Electronic Resource, http://www.nanometer.ru/2009/02/ 12/biosensori_58480.html.

5. Emory, J.M. and Soper, S.A., Anal. Chem., 2008, vol. 80, no. 10, p. 3897.

6. Lange, J.J., Culbertson, C.T., and Higgins, D.A., Anal. Chem., 2008, vol. 80, no. 24, p. 9726.

7. Gao Chanlu, Ging Shu, Tan Qiulin, and Gu Li-Qun, Anal. Chem., 2009, vol. 81, no. 1, p. 80.

Yu. A. Zolotov 